\title{
Assessing the effects of cognitive ageing on navigation: a novel navigation test battery
}

Jan M. Wiener ${ }^{1}$, Denise Carroll', Stacey Juthapan ${ }^{1}$, Iram Bibi ${ }^{1}$, Dima Ivanova ${ }^{1}$, Thomas Wolbers ${ }^{2}$

${ }^{1}$ Department of Psychology, Ageing and Dementia Research Centre, Bournemouth University, Poole, UK

${ }^{2}$ German Centre for Neurodegenerative Diseases, Magdeburg, Germany 


\begin{abstract}
Most research groups studying human navigation behaviour with virtual environments (VE) technology develop their own paradigms. This makes it difficult to compare results between groups, but also to create normative data sets for any specific navigation task. Such norms, however, are prerequisites for the use of navigation assessments as diagnostic tools, for example, to support the early and differential diagnosis of atypical aging. Here we start addressing these problems by presenting and evaluating a new navigation test battery that we make freely available to other researchers (https://osf.io/mx52y/). Specifically, we designed three navigation tasks, which are adaptation of earlier published paradigms used to study the effects of typical and atypical ageing on navigation: a route repetition task that can be solved using egocentric or viewpoint dependent navigation strategies, and a route retracing and the different approach direction tasks both of which require viewpoint independent processing. Despite introducing a number of changes to the original paradigms to make them look realistic and ecologically valid, and therefore easy to explain to people unfamiliar with VE or with cognitive impairments, we were able to replicate the findings from the original studies. Specifically, we found general age-related declines in navigation performance and additional specific deficits in tasks that required viewpoint independent or allocentric processes. These findings demonstrate that our new paradigms render reliable results and are thus suited to be used more widely.
\end{abstract}

\title{
Introduction
}

A growing number of studies have demonstrated age-related declines in a variety of orientation and navigational tasks, including route learning and repetition (Head \& Isom, 2010; Zhong \& Moffat, 2016), route retracing (Wiener et al., 2012), cognitive mapping (Moffat \& Resnick, 2002) and wayfinding (laria et al., 2009). However, the rise of, and relatively easy access to, virtual environments technology means that most research groups design and develop their own navigation paradigms, which makes it difficult to compare findings from different research groups. As a consequence, we do not have normative data even for the most fundamental or frequent navigation tasks, such as learning to navigate a novel route. Here we begin to address these problems by developing and evaluating three different navigation tasks which (1) are based on established and published earlier navigation paradigms (Waller \& Lippa, 2007; Head \& Isom, 2010; Wiener et al., 2012 , 2013), (2) which are easy to amend to address specific research questions, and (3) which we make freely available to other research groups (https://osf.io/mx52y/). 
Spatial disorientation is one of the earliest signs of atypical aging, particularly in cases of Alzheimer's Disease (AD; Pai \& Jacobs, 2004; Serino et al., 2015). It has even been argued that middle aged adults at a high risk of developing AD show poorer spatial abilities (Ritchie et al., 2017) and that spatial memory tasks predict conversion from Mild Cognitive Impairment (MCl) to AD (Wood et al., 2016). A likely explanation for the sensitivity of spatial tasks and navigation tasks for atypical aging is that brain areas involved in navigation, in particular the medial entorhinal cortex (EC) and the precuneus, show presymptomatic AD-induced pathology (Khan et al., 2015; Weston et al., 2016). Assessments of navigation abilities therefore have the potential to become powerful diagnostic tools to support the early and differential diagnosis of atypical aging. However, in order to do so, normative data for a series of established navigation tasks which rely on different navigation mechanisms are needed.

Declines in orientation and navigation abilities (starts same as paragraph above, maybe we can invert sentence) in both typical and atypical aging have now been well documented (for a recent review, see Lester et al., 2017). Importantly, successful navigation is based on different navigation strategies and mechanisms, which decline at different rates. Specifically, egocentric navigation strategies, also often referred to as route learning or response strategies, are typically preserved for longer than allocentric strategies, which require the processing and encoding of the spatial relationship between landmarks and/or places (Moffat, 2009).

Here we designed three different navigation tasks which are based on navigation tasks used in earlier aging studies. The first task is a route repetition task. Route navigation belongs to the most frequent human navigation tasks and route knowledge is typically conceptualised as a series of stimulus-response associations in which places or landmarks become associated with movement directions (e.g., "Turn left at gas station", Waller \& Lippa, 2009). In the absence of landmarks, routes can be remembered simply as a sequence of movement instructions (e.g., left, right, straight, right, left; Waller \& Lippa, 2009). Importantly, both of these strategies rely on spatial information encoded in an egocentric reference frame (Wolbers $\&$ Wiener, 2014). Route navigation has been suggested to be supported by the striatal circuit (Hartley et al., 2003). Several studies have addressed how cognitive aging affects route learning and knowledge. While most studies report age-related declines in route earning abilities (Head \& Isom, 2010), recent studies suggest that age-related differences in route knowledge - such as knowledge about the specific associations between landmarks and directions - can be ameliorated if both age groups are trained to criteria, which takes longer in the older age group (O'Malley et al., 2017). 
The second task is a route retracing task. In contrast to route repetition, route retracing refers to navigating a route from the end point back to the start point. In route retracing decision points are approached from a viewpoint different to that experienced during encoding. Simple S-R associations therefore do not support route retracing. Rather, route retracing requires knowledge about the spatial relationship between the direction from which a decision point is approached and the direction in which the route proceeded. Such representations are viewpoint-independent, or allocentric. To our knowledge only a single study has addressed the effects of cognitive aging on route repetition and route retracing (Wiener et al., 2012). While younger participants outperformed the older age group in both route repetition and route retracing, the performance differences were particularly pronounced in retracing and in contrast to the repetition condition, older adults did not learn in the retracing condition over the course of the experiment.

The third task, the Different Approach Direction task, is an adaptation of a paradigm developed by Wiener et al. (2013). In this task, participants had to recall from which street they originally approached an intersection, when seeing it from a viewpoint they had not experienced before. Solving this task requires participants to encode the configuration of landmarks at the intersection in relation to the direction from which this intersection was approached originally. While younger participants adopted an allocentric strategy to solve this task over the course of the experiment, older adults failed to adopt the correct strategy and showed a consistent maladaptive bias for an egocentric strategy.

When designing the new tasks, we aimed to ensure that the environments looked realistic, and that the tasks were ecologically valid and easy to explain. These design considerations are important when working with older adults who are not used to virtual environments and with people with cognitive impairments to ensure that they easily understand the tasks.

The primary aim of the current study was to introduce and evaluate a new virtual environment navigation test battery which builds on earlier research. Despite adapting the paradigms as outlined above, we expected to replicate earlier findings. Specifically, we expected that our younger participant group would outperform our older participant group in all three tasks (Head \& Isom, 2010; Wiener et al., 2012, 2013). We expected route repetition performance to be higher than route retracing performance, and we expected older adults to show impaired learning in the route retracing task as compared to younger participants (Wiener et al., 2012). We expected performance in the different approach direction task to rely on the amount of misalignment between 
the encoding and the test perspective (de Condappa \& Wiener, 2016) and our older age group to be more strongly affected by larger misalignments than our younger age group (Watanabe, 2011).

In contrast to earlier studies, we tested our participants on all three tasks, which allowed us to relate performance between tasks. We expected navigation tasks that share more cognitive mechanisms or strategies to be more strongly correlated than tasks that share fewer cognitive mechanisms or strategies. Specifically, it has been suggested that both the route retracing and the different approach direction task require viewpoint independent processing (Wiener et al., 2012; de Condappa \& Wiener, 2016), while route repetition relies on egocentric, viewpoint dependent strategies (Waller \& Lippa, 2010). We therefore expected performance between the route retracing and the different approach direction tasks to be more strongly correlated than performance between the route repetition task and the route retracing or different approach direction task.

\section{Materials and methods}

\section{Participants}

Eighty-one participants were recruited from Bournemouth University (BU) and the surrounding area. These comprised 37 younger participants aged 18-32 (23 females, mean age: $20.57 \pm 3.00$ ), and 44 older participants aged 60-82 (29 females, mean age: $71.02 \pm 5.50$ ). All participants had normal or corrected to normal vision. BU students received credits for their contribution to the project, and external participants received $£ 12$ to cover their expenses. To screen for cognitive impairments, we administered the ACE III (Addenbrooke's Cognitive Examination; Mathuranath et al., 2000). All participants scored above the threshold of $82 / 100$ suggestive of cognitive impairments (Hsieh et al., 2013; Noone, 2015). The mean ACE-III score for the younger participant group was 92.30 and 95.98 for the older participant group.

\section{The Navigational Test Battery}

Based on earlier paradigms, we developed a novel navigation test battery that consisted of three navigation tasks; the route repetition task, the route retracing task and the different approach direction task. We used UNITY (https://unity3d.com/) to program the navigation test battery. All tasks made use of the same virtual environment, which consisted of streets and four-way intersections in a residential neighbourhood. The houses bordering the streets were all identical (see Figure 1). Depending on the navigation task (explained in more detail below), we introduced unique 
houses (i.e. landmarks) to the intersections. The Navigation Test Battery can be obtained for free from https://osf.io/mx52y/.

\section{Route Repetition task}

The route repetition task is a typical route learning task, designed to assess participants' ability to learn an unfamiliar route and to repeat this route in the same direction as during training. (e.g., Waller \& Lippa, 2007). It was composed of three experimental blocks, each of which featured a training phase and a test phase.

At the beginning of the training phase, participants were positioned in a street next to a black car (see Figure 1 top left). They were then passively transported along a route that was comprised of six intersections with two left turns, two right turns and two straight movements. The route stopped at a red phone box (Figure 1 top right). Each intersection featured four identical houses (landmarks) positioned at the four corners of the intersection (see Figure 1 top middle). Different intersections featured different houses, such that each intersection could be unambiguously identified. Participants were instructed to learn the route during the training phase.

In the test phase, participants were asked to reproduce the route from the car to the phone box, one intersection at a time. The first test trials started at the car, the other test trials started in the centre of an intersection, such that the houses at the far end of the current intersection were visible. Participants were then transported passively towards the next intersection and stopped just before entering the intersection. They were then prompted to indicate the direction in which the route continued. After participants gave their response, by pressing the corresponding arrow key, they were teleported to the centre of the intersection, facing the street that led to the next intersection along the route. In other words, participants did not receive feedback during the test phase. There were no time constraints. To assess learning, we presented the same route in each of the three experimental sessions. 

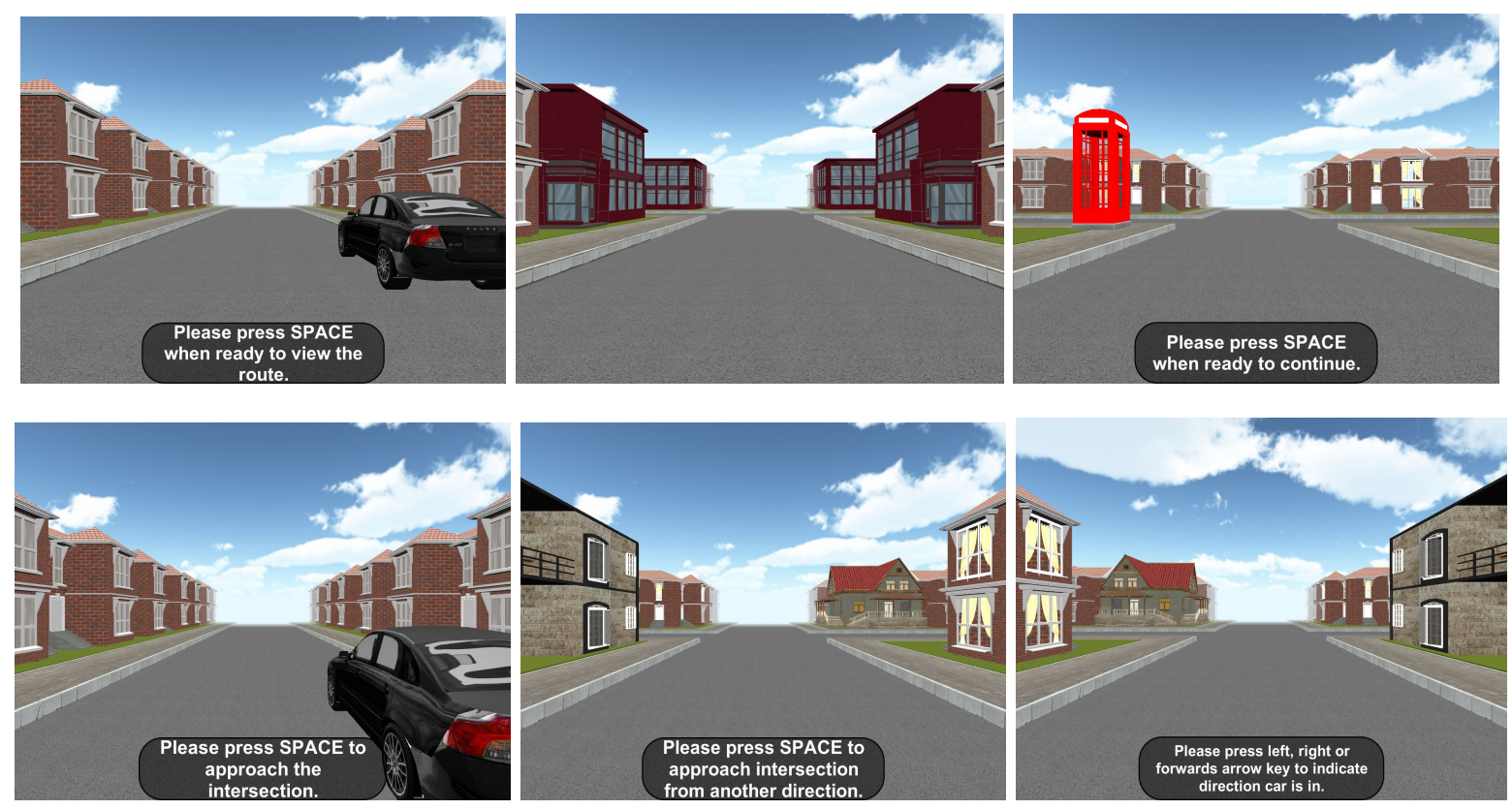

Figure 1: Top row: Screenshots taken during the training phase of the route repetition or route retracing task; bottom row: screenshots taken during the different approach direction tasks.

Participants could use two strategies to learn the route, a sequence of direction strategy (here: left, right, straight, right, left, straight) and/or an associative cue strategy in which they associate a direction with a particular landmark ("Turn right at Blue House"; Waller and Lippa, 2007). The current task was not designed to investigate how these strategies interact during route learning, but the Navigational Test Battery (https://osf.io/mx52y/) does allow presenting the intersections in random order during the test phase, in which case participants cannot rely on a sequence of turn strategy.

\section{Route Retracing Task}

The route retracing task was designed to assess participants' ability to find their way back to the starting point after being transported along a route. In earlier work, we have argued that such route repetition behaviour cannot be explained by simply mirroring egocentric route knowledge when retracing the route. Rather, successfully retracing a route requires allocentric processing (Wiener et al., 2012).

The route retracing task was identical to the route replicate task, with the exception that participants, in the test phase, had to find their way back from the phone box to the black car. In other words, participants had to navigate the route in the opposite direction as compared to the training phase. 


\section{Different Approach Direction Task}

The different approach direction task was designed to test participants' ability to encode the configuration of houses (landmarks) relative to the street in which the car was parked. The paradigm is based on a similar task and is thought to assess allocentric processing and perspective taking abilities (Wiener et al., 2013; de Condappa \& Wiener, 2016).

The different approach direction task consisted of 18 independent trials. Each trial began with an encoding phase: participants were positioned in a street next to a black car (see Figure 1 bottom left); they were then passively navigated towards a single intersection that featured two unique houses (i.e. landmarks) at diagonally opposite corners of the intersection (see Figure 1 bottom middle). Movement stopped just before the intersection was entered, such that both unique houses were in view. The participants' task was to memorise were their car was parked.

In the test phase, participants were passively transported toward the same intersection, but from one of the other three streets (see Figure 1 bottom right). Movement stopped just before they entered the intersection and participants were asked to indicate the direction in which the car was parked, i.e. to indicate the street from which they approached the intersection during the encoding phase.

The car was always parked in the street to the south of the intersection (see Figure 2). During the test phase, participants approached the intersection either from the street to the west, to the north or to the east. Importantly, the perspective shifts required to align the view during the test phase with that experienced during the encoding phase was larger when approaching the intersection from the north $\left(180^{\circ}\right)$ as compared to the east $\left(90^{\circ}\right)$ or west $\left(90^{\circ}\right)$. Note that participants did not know about these cardinal directions in the experiment - we are simply using them for the purpose of presenting the paradigm and the data. 

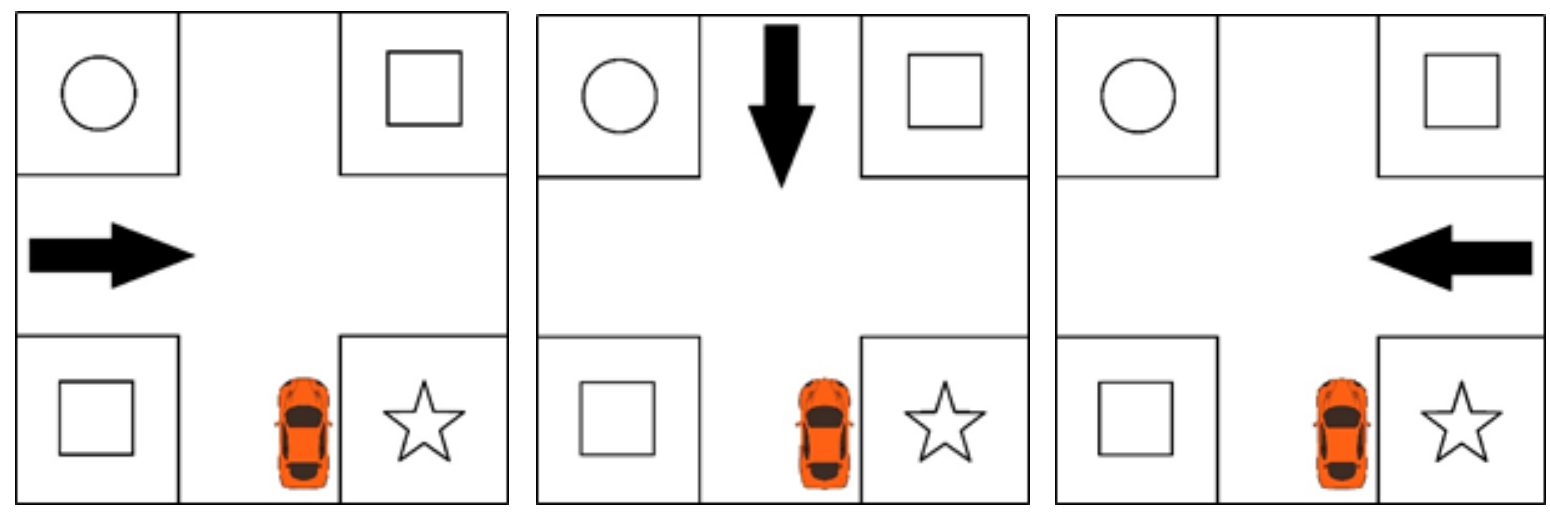

Figure 2: Schematic drawing illustrating the three different test situations in the different approach direction task. During the encoding phase, participants always approached the intersection from street to the south. During the test phase, they approached the same intersection either from the street to the east, west, or north. Note that approaches to the intersection from the east or west were misaligned with the encoding situation by $90^{\circ}$, while an approach

from the north was misaligned with the encoding situation by $180^{\circ}$

There were 18 trials in the different approach direction task, each with a unique combination of houses (landmarks) at the intersection. Six trials required a left response, six trials required a right response, and six trials required a straight on response. The trials were presented in random order. A single trial ended after participants made their response and participants did not receive any feedback. Note that, in contrast to the route repetition and route retracing task, the different approach direction task did not require participants to learn a route with multiple decision points. The different approach direction task therefore had much lower long term memory involvement than the other tasks.

Each task began with a short demo to help participants to understand what was required of them. Note also, that we introduced white fog to the environment for all three experimental tasks. This ensured that participants could only see one intersection at any time.

\section{Procedure}

Participants came to the Psychology Department at BU for one 90 minute test session. They began by completing the ACE-III cognitive assessment to ensure that they scored above the cut-off value $(82 / 100)$ and were eligible to continue with the study. None of the participants were excluded on this basis. Following this, participants completed the three navigational tasks of the Navigational Test Battery on a computer with a 21" screen. We balanced the order in which the navigation tasks 
were administered between participants and age groups to ensure that there were no systematic biases introduced by potential order effects.

\section{Results}
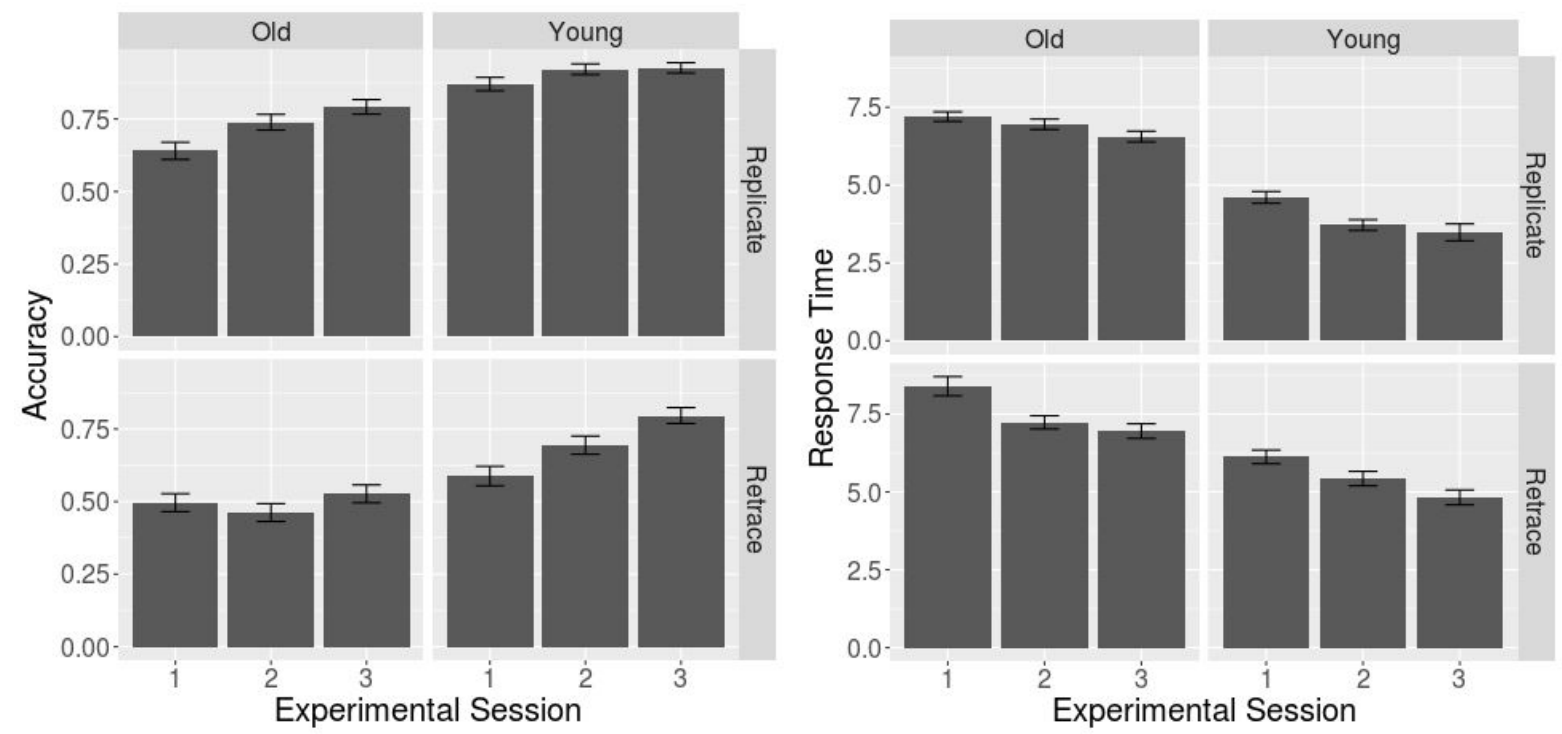

Figure 3: Performance (left panel) and response times (right panel) for our older and younger participant group in the Replication and Retrace task as a function of experimental sessions.

\section{Route repetition and route retracing}

\section{Performance}

A repeated measures ANOVA with the within factors task (replicate, Retrace) and session (1, 2 , and 3 ) and the between factors age group (young, old), and gender (male, female) revealed significant main effects of task $\left(F_{(1,76)}=53.29, p<0.001\right)$, block $\left(F_{(2,152)}=10.23, p<0.001\right)$, and of age group $\left(F_{(1,76)}=27.99, p<0.001\right.$, $)$, but not of gender $\left(F_{(1,76)}=2.54, p=0.11\right)$. Specifically, participants performed better in the replication than the retrace task, younger participants performed better than older participants and performance in the third experimental session was greater than in the first experimental session $(p<0.01$, see Figure 3$)$.

Only one interaction, the interaction between task, block, and age-group was significant $\left(F_{(2,152)}=4.85, p=0.01\right)$. To further explore the nature of this three way interaction we carried out 
separate ANOVAs for the repetition and the retrace task. We found a significant effect of age group for both tasks (Repetition: $F_{(1,78)}=16.5 s 3, p=0.001$; Retrace: $F_{(1,78)}=16.09, p=0.001$ ), demonstrating that the younger group performed better than the older group in both tasks. In both tasks there was an effect of session (Repetition: $F_{(2,156)}=8.44, p<0.01$; Retrace: $F_{(2,156)}=4.65, p=0.01$ ) with better performance in later experimental sessions. In the Repetition task the interaction between age group and experimental session was not significant $(p=0.2)$. In the Retrace task, in contrast, the interaction between age group and experimental session was significant $\left(F_{(2,156)}=3.09, p<0.05\right)$. This interaction was driven by a significant increase in performance in the young participant group between block 1 and block 3 ( $p<0.01$ ), while the older age group did not significantly improve over the course of the experiment (block 1 vs block 3: $p=0.61$ ). These additional analyses suggest that the significant three-way interaction between age group, task, and block in the original ANOVA was driven by significant learning effect in the young participants group in the Retrace task which was absent in the older participant group (see Figure 3).

\section{Response Time}

A repeated measures ANOVA with the between factors age group (young, old) and gender (male, female) and the within factors task (repetition, retrace) and experimental session $(1,2,3)$ revealed significant main effects of age group $\left(F_{(1,76)}=31.70, p<0.001\right)$, task $\left(F_{(1,76)}=20.44, p<\right.$ $0.001)$ and experimental session $\left(F_{(1,76)}=39.16, p<0.001\right)$, but not of gender $\left(F_{(1,76)}=2.00, p=0.16\right)$. Response time was longer in the old age group than in the young age group, longer for the retrace task than the repetition task and decreased over experimental sessions. None of the interactions were significant.
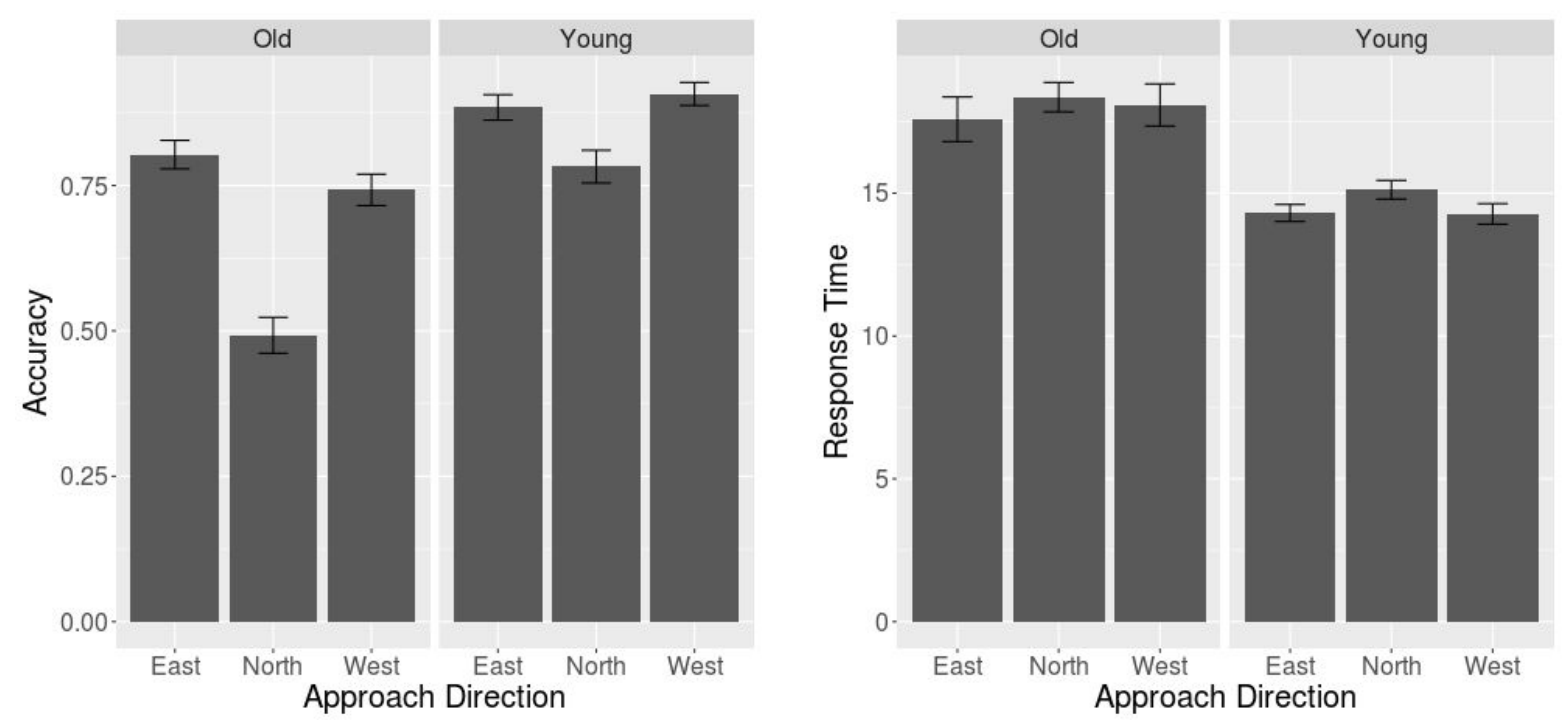
Figure 4: Performance (left panel) and response times (right panel) for the older and younger participant group in the different approach direction task. Note that approaching the intersection from the East or West was misaligned with the encoding situation by $90^{\circ}$, while approaching the intersection from the North was misaligned with the encoding situation by $180^{\circ}$.

\section{Different Approach Direction Task}

A repeated measures ANOVA with the within factor Approach Direction (West, North, South) and the between factors age group (young, old), and gender (male, female) revealed significant main effects of approach direction $\left(F_{(2,152)}=28.13, p<0.001\right)$ and age group $\left(F_{(1,76)}=15.21, p<0.001\right)$, but not of gender $\left(F_{(1,76)}=3.21, p=0.08\right)$. Specifically, younger participants performed better than older participants, and performance for an approach from North was significantly worse than performance for either an East $(p<0.001)$ or West $(p<0.001)$ approach. Performance for West and East approach was similar $(p=0.33)$. Only the interaction between age group and approach direction rendered a significant result $\left(F_{(2,152)}=5.29, p<0.01\right)$. This interaction was driven by a stronger decline in performance in the older participant group as compared to the young participant group, when approaching the intersection from the North arm than from the East or West arm $(p=0.01)$.

To investigate whether performance increased over the course of the 18 trials, we calculated correlations between performance and trial number (1-18). Neither when combining young and old participants ( $r=0.36, p=0.15$ ), nor for any age group independently (young: $r=0.39, p=0.11$; old: $r$ $=-.25, p=0.32$ ), did we find significant correlations, suggesting that participants performance did not significantly improve over trials.

\section{Response Time}

A repeated measures ANOVA with the within factor Approach Direction (West, North, South) and the between factors age group (Young and Old), and gender (male, female) revealed a significant main effect only of age group $\left(F_{(1,76)}=11.97, p<0.001\right)$ and did not reveal any significant interactions. Specifically, response time was longer for the old age group than the young age group.

\section{Correlations between navigation tasks}

As all participants completed all three navigation tasks (different approach direction, route retracing, route repetition), we were able to correlate performance between the different tasks. We 
predicted that we would find stronger correlations between the route retracing and the different approach direction task, both of which have been suggested to require some viewpoint independent processing (Wiener et al., 2012, 2013), as compared to the route repetition and the route retracing or the different approach direction task, where the former can be solved with purely egocentric and viewpoint dependent strategies. To control for the differences in overall performance between the older and younger participants presented above, we first normalised the data by subtracting the mean of each task and each age group before combining data from old and young participants. As predicted, we found a stronger correlation between the different approach direction and the route retracing task $(r=0.35, p<0.01)$ than between the route repetition and the route retracing task $(r=$ $0.17, p=0.13$ ) or between the different approach direction task and the route repetition task $(r=$ $0.25, p=0.03)$, even though the latter correlation was significant.

\section{Discussion}

The main aim of the current study was to introduce and evaluate three new navigation tasks. Based on earlier published work, we present tasks addressing the ability to learn a route, the ability to retrace a recently travelled path, and the ability to learn and use a configuration of landmarks. When designing these tasks, we aimed to make them ecologically valid, and easy to explain and administer, such that they would be suitable for use with older adults and patient groups in the future.

The design of the route repetition task was based on earlier research (e.g., Head \& Isom, 2010). In each of three experimental sessions, participants were passively navigated along a route featuring six intersections starting from a parked car and ending at a phone box. In the test phase, participants were navigated towards each intersection in the same order as during training and were asked to indicate the direction in which the route continued. We were able to replicate earlier findings (Head \& Isom, 2010). Specifically, while both our young and our older participant group showed learning over the course of the experiment, the young participant group performed better than the old participant group. Analyses of response times, which show longer response times in the older age group (Salthouse, 1996), suggest that the age-related decline in route learning abilities cannot be explained by a speed accuracy trade-off.

In the current route learning paradigm, participants can use two strategies to solve the task, an associative cue strategy in which they associate landmarks with movement directions and a sequence of turn strategy, in which they remember the sequence of turns required to navigate the route (Waller \& Lippa, 2007). While it is beyond the scope of this study to investigate the 
interrelation between these strategies, it is important to point out that both may be affected by aging. Firstly, the associative cue strategy relies on associative learning which is affected by cognitive aging (Naveh-Benjamin et al., 2003). Secondly, the learning of sequences of turns along a route relies on the hippocampal circuit (Igloi et al., 2010), which undergoes substantial functional and structural changes already during the typical aging process (Fjell et al., 2013). Current research in our laboratory aims to disentangle the contribution of the associative cue and the sequence of turns strategy and the effect that cognitive aging has on each strategy.

The route retrace paradigm was identical to the route repetition paradigm discussed above, but required participants to navigate from the end of the route back to the start location. This is a frequent real-world navigation task, for example when exploring an unfamiliar part of a city, that has received very little attention in the literature. The paradigm used here was inspired by earlier work (Wiener et al., 2012), and while the exact procedure was slightly different to the one we used earlier, we were able replicate the main findings: first, our young participants outperformed our older participants; second, we found that our young, but not our old, participant group improved over the course of the experiment.

It is important to note that egocentric strategies, such as the associative cue strategy ("Turn left at church") or the sequence of turn strategy discussed above, do not directly support route retracing, as intersections are approached from a different direction than during the encoding of the route. While it is conceivable, that participants would simply mirror the direction of turn required at each intersection when retracing a route, this does not explain why our older age group did not show any learning over experimental sessions. Moreover, if route repetition and route retracing relied on the same cognitive strategies, we expected a correlation between performance in these tasks, which we have not found. In contrast, we found a significant correlation between the route retracing and the different approach direction task (discussed in more detail below). This is in line with the explanation that route retracing requires abstracting from a viewpoint-dependent representation which can be achieved by encoding the spatial relationship between the street from which an intersection was approached and the street in which the route proceeded (Wiener et al., 2012). The encoding and processing of such viewpoint-independent, or allocentric, representations are affected by cognitive aging (Moffat and Resnick, 2002; Moffat et al., 2007, Harris \& Wolbers, 2012), possibly resulting from age-related hippocampal neurodegeneration (Raz et al., 2010).

The different approach direction task required participants to encode the spatial relationship of landmarks at an intersection and the direction or street from which this four-way intersection was 
approached. In the test phase, participants then approached the same intersection from any of the remaining three directions/streets. The paradigm was based on earlier work (Wiener et al., 2013) which showed age-related declines in participants ability to channel into a route when approaching an intersection from a different direction than during training. In contrast to the earlier paradigm, participants were not required to learn a route in the current study, but only the direction from which they approached a single intersection during training. Despite these methodological differences, we found an age-related decline in participants ability to solve the task. Importantly, we found an interaction between approach direction and age group with older adults showing stronger performance decrements when the approach direction in the test phase was misaligned with the encoding situation by 180 instead of 90 degrees. This corroborates earlier notions that the task requires perspective shift to align the current viewpoint with the encoded viewpoint (de Condappa \& Wiener, 2016), a process which is affected by cognitive aging (Watanabe, 2011). Finally, we did not find a significant correlation between the trial number and performance in the different approach direction task which suggests that this task did neither benefit from or require training.

Taken together, we have introduced here a new experimental software package for three navigation tasks, which are adaptations of earlier paradigms (Head \& Isom, 2010; Wiener et al., $2012,2013)$. The new navigation tasks presented here were carefully designed such that they (i) resemble real world navigation situations more closely than the original tasks, (ii) that they are easy to explain to participant groups who may have little experience with virtual environments technology, and (iii) that they are easy to modify to address other research questions or to test other participant groups. For example, our young participant group showed very strong performance in the route repetition task. To further study the route learning in young participants, it would be easy to lengthen the route or, to address the interaction of the associative cue and the sequence of turn strategy (Waller \& Lippa, 2010), to present the intersection in random order during the test phase. Importantly, and despite adapting the original paradigms, we replicated their main findings which demonstrates that the new navigation test battery introduced here renders reliable results. By making the test battery freely available to other research groups (https://osf.io/mx52y/), we hope to contribute to the development of normative data sets which are crucial for the development of navigation assessments in a clinical context.

\section{Acknowledgements}

Thanks to Peter Allen for his help in programming the navigation test battery. 


\section{References}

de Condappa, O., \& Wiener, J. M. (2016). Human place and response learning: Navigation strategy selection, pupil size and gaze behavior. Psychological Research, 80(1), 82-93.

Fjell, A.M., McEvoy, L., Holland, D., Dale, A. M., \& Walhovd, K. B. (2013). Brain Changes in Older Adults at Very Low Risk for Alzheimer's Disease. The Journal of Neuroscience, 33(19), 8237-8242.

Harris MA, Wolbers T (2012) Ageing effects on path integration and landmark navigation. Hippocampus 22: $1770-1780$.

Hartley, T, Maguire, EA, Spiers, HJ, Burgess, N (2003). The well-worn route and the path less traveled: distinct neural bases of route following and wayfinding in humans. Neuron, 37, 5:877-88.

Head, D, Isom, M (2010). Age effects on wayfinding and route learning skills. Behav. Brain Res., 209, 1:49-58.

Hsieh, S., Schubert, S., Hoon, C., Mioshi, E., \& Hodges, J. R. (2013). Validation of the Addenbrooke's Cognitive Examination III in frontotemporal dementia and Alzheimer's disease. Dementia and geriatric cognitive disorders, 36(3-4), 242-250.

laria, G., Palermo, L., Committeri, G. \& Barton, J. J.S. (2009). Age differences in the formation and use of cognitive maps. Behavioural Brain Research, 196(2), 187-191.

Iglói, K, Doeller, CF, Berthoz, A, Rondi-Reig, L, Burgess, N (2010). Lateralized human hippocampal activity predicts navigation based on sequence or place memory. Proc. Natl. Acad. Sci. U.S.A., 107, 32:14466-71.

Khan, UA, Liu, L, Provenzano, FA, Berman, DE, Profaci, CP, Sloan, R, Mayeux, R, Duff, KE, Small, $S A$ (2014). Molecular drivers and cortical spread of lateral entorhinal cortex dysfunction in preclinical Alzheimer's disease. Nat. Neurosci., 17, 2:304-11.

Lester, AW, Moffat, SD, Wiener, JM, Barnes, CA, Wolbers, T (2017). The Aging Navigational System. Neuron, 95, 5:1019-1035.

Mathuranath, P. S., Nestor, P. J., Berrios, G. E., Rakowicz, W., \& Hodges, J. R. (2000). A brief cognitive test battery to differentiate Alzheimer's disease and frontotemporal dementia. Neurology, 55(11), 1613-1620.

Moffat SD, Resnick SM (2002) Effects of age on virtual environment place navigation and allocentric cognitive mapping. Behav Neurosci 116:851-859

Moffat, SD, Kennedy, KM, Rodrigue, KM, Raz, N (2007). Extrahippocampal contributions to age differences in human spatial navigation. Cereb. Cortex, 17, 6:1274-82.

Moffat, SD (2009). Aging and spatial navigation: what do we know and where do we go?. Neuropsychol Rev, 19, 4:478-89.

Naveh-Benjamin, M., Hussain, Z., Guez, J., \& Bar-On, M. (2003). Adult age differences in episodic memory: Further support for an associative-deficit hypothesis. Journal of Experimental Psychology: Learning, Memory, and Cognition, 29(5), 826-837.

Noone, P. (2015). Addenbrooke's Cognitive Examination-III. Occupational Medicine, 65(5), 418-420.

O’Malley, M., Innes, A. \& Wiener, J.M. Mem Cogn (2017). https://doi.org/10.3758/s13421-017-0763-7

Pai, M. C., \& Jacobs, W. J. (2004). Topographical disorientation in community-residing patients with Alzheimer's disease. Int J Geriatr Psychiatry, 19(3), 250-252.

Raz N, Lindenberger U, Rodrigue KM,Kennedy KM, Head D, Williamson A, Dahle C, Gerstorf D, Acker JD (2005) Regional brain changes in aging healthy adults: general trends, individual differences and modifiers.Cereb Cortex 15:1676-1689. 
Ritchie, K, Carrière, I, Su, L, O'Brien, JT, Lovestone, S, Wells, K, Ritchie, CW (2017). The midlife cognitive profiles of adults at high risk of late-onset Alzheimer's disease: The PREVENT study. Alzheimers Dement, 13, 10:1089-1097.

Salthouse, TA (1996). The processing-speed theory of adult age differences in cognition. Psychol Rev, 103, 3:403-28.

Serino, S., Morganti, F., Di Stefano, F. \& Riva, G. (2015). Detecting early egocentric and allocentric impairments deficits in Alzheimer's disease: An experimental study with virtual reality. Frontiers in Aging Neuroscience, 7(88). DOI: 10.3389/fnagi.2015.00088.

Waller, D., \& Lippa, Y. (2007). Landmarks as beacons and associative cues: their role in route learning. Memory \& Cognition, 35(5), 910-924.

Watanabe, M (2011). Distinctive features of spatial perspective-taking in the elderly. Int J Aging Hum Dev, 72, 3:225-41.

Weston, PS, Nicholas, JM, Lehmann, M, Ryan, NS, Liang, Y, Macpherson, K, Modat, M, Rossor, MN, Schott, JM, Ourselin, S, Fox, NC (2016). Presymptomatic cortical thinning in familial Alzheimer disease: A longitudinal MRI study. Neurology, 87, 19:2050-2057.

Wiener, J. M., Kmecova, H., \& de Condappa, O. (2012). Route repetition and route retracing: effects of cognitive aging. Frontiers in Aging Neuroscience, $4,7$.

Wiener, J. M., de Condappa, O., Harris, M. A., \& Wolbers, T. (2013). Maladaptive bias for extrahippocampal navigation strategies in aging humans. The Journal of Neuroscience, 33(14), 6012-6017.

Wood, RA, Moodley, KK, Lever, C, Minati, L, Chan, D (2016). Allocentric Spatial Memory Testing Predicts Conversion from Mild Cognitive Impairment to Dementia: An Initial Proof-of-Concept Study. Front Neurol, 7:215.

Zhong, J. Y., \& Moffat, S. D. (2016). Age-Related Differences in Associative Learning of Landmarks and Heading Directions in a Virtual Navigation Task. Frontiers In Aging Neuroscience, 8(122). https://doi.org/10.3389/fnagi.2016.00122 